\title{
NM23 et les gènes suppresseurs de métastases
}

> La dissémination métastatique est la cause majeure des décès dus au cancer. Les mécanismes capables de conférer à une cellule tumorale un pouvoir métastatique sont complexes et encore mal connus. Plusieurs hypothèses sont proposées dont l'existence de cellules à potentiel métastatique dès les premières étapes de la formation de la tumeur primitive. Ce potentiel serait dû à la dérégulation de gènes spécifiques capables de favoriser ou d'inhiber la dissémination métastatique. Ainsi, les GSM, «gènes suppresseurs de métastases », objets de cet article, sont définis par leur capacité à supprimer in vivo le développement des métastases sans affecter la croissance de la tumeur primitive. La plupart des GSM modulent les voies de signalisation mettant en jeu les protéines $G$ et la voie des MAP-kinases. Chez l'homme, plusieurs études cliniques ont établi une corrélation inverse entre l'expression de ces gènes dans la tumeur primitive et la survie des patients. Les GSM modulent le potentiel métastatique d'une tumeur essentiellement via des mécanismes épigénétiques, ce qui suggère qu'une réactivation de leur expression endogène pourrait constituer une voie thérapeutique anti-métastatique prometteuse. Nous présenterons dans cette synthèse les différents GSM connus actuellement, tout particulièrement NME (NM23), objet de nos recherches, ainsi que les voies de signalisation dans lesquelles ces gènes sont potentiellement impliqués. <

Près de $90 \%$ des décès des patients atteints de cancer sont dus aux métastases. Les mécanismes moléculaires précis impliqués dans la dissémination métastatique sont mal connus et leur compréhension devrait faire considérablement progresser la thérapie anti-cancéreuse. L'existence de gènes contrôlant spécifiquement le potentiel métastatique a été rapportée pour la première fois avec la découverte du gène NM23 [1].

\section{Mathieu Boissan, Marie-France Poupon, Marie-Lise Lacombe}

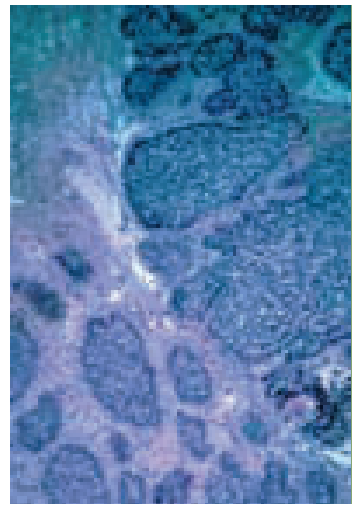

M. Boissan, M.L. Lacombe

Inserm, U680, Paris, F-75012 ;

Université Pierre et Marie Curie-

Paris, F-75005, France.

Faculté de Médecine UMRS 680,

27 , rue Chaligny,

75012 Paris, France.

M.F. Poupon : Inserm, U612,

Institut Curie, Paris,

F-75005, France.

lacombe@st-antoine.inserm.fr

II s'agit de gènes suppresseurs de métastases, objets de cet article, mais il existe aussi des gènes dont les produits favorisent le processus métastatique, et qui pourraient intervenir de façon positive sur les mêmes voies.

La formation d'une métastase («métastatogenèse »), tumeur secondaire à distance de la tumeur primitive, nécessite que les cellules tumorales franchissent de nombreuses étapes (Figure 1): (1) le détachement de la tumeur primitive; (2) l'envahissement du stroma péritumoral (invasion); (3) l'entrée (intravasation) et la survie dans la circulation sanguine et/ou lymphatique; (4) l'arrêt au niveau de l'endothélium d'un organe cible secondaire; (5) l'extravasation dans le tissu environnant; (6) la prolifération locale au niveau du site secondaire ou colonisation métastatique conduisant à la formation des métastases cliniquement détectables appelées macrométastases. Cependant, la présence de cellules tumorales disséminées au niveau du site secondaire ne permet pas de prédire l'apparition de métastases. En effet, les cellules tumorales peuvent être présentes mais rester quiescentes (état de dormance tumorale). Ces cellules tumorales dormantes peuvent évoluer sous forme de macrométastases de nombreuses années après un traitement apparemment efficace de la tumeur d'origine. La cascade métastatique dépend des propriétés intrinsèques des cellules cancéreuses et de leur interaction avec les cellules stromales, et de la capacité du micro-environnement tumoral à fournir une « niche » pour leur implantation. De multiples voies de signalisation modifiant les interactions cellule/cellule et cellule/matrice extra-cellulaire, et influençant les propriétés adhésives, invasives et migratoires des cellules tumorales, sont mises en jeu dans la cascade métastatique [2, 3, 44].

Plusieurs hypothèses pourraient expliquer la formation des métastases [4]. La première, ancienne, propose l'existence de sous-populations de cellules tumorales, variantes et rares, ayant acquis un avantage sélectif pour la dissémination métastatique. La deuxième, plus récente et obtenue à partir de l'observation des tumeurs des patients, suggère la présence d'une 
prédisposition métastatique établie dès le développement de la tumeur primitive. La recherche d'une «signature métastatique » a été possible grâce aux progrès de la génomique fonctionnelle. Les analyses d'échantillons de tumeurs humaines par micro-arrays ont montré que certaines combinaisons d'altérations de l'expression génique induiraient une croissance tumorale sans propension à métastaser alors que d'autres permettraient le développement d'une tumeur à potentiel métastatique. II est intéressant de noter que plusieurs voies impliquées dans le potentiel métastatique (SRC, PI3K, MAPK...) sont également activées lors des processus oncogéniques suggérant des mécanismes communs. Cependant, la découverte que certains gènes, par leur absence ou leur présence, favorisaient ou inhibaient la dissémination métastatique, indique que ce processus peut être contrôlé spécifiquement. Les gènes suppresseurs de métastases (GSM) ont été identifiés par la diminution de leur expression dans des cellules tumorales métastatiques par rapport aux cellules tumorales non métastatiques à l'aide de techniques telles que le criblage différentiel, l'hybridation soustractive, les puces à ADN ou le transfert cellulaire de chromosomes (Tableau I). Ces gènes sont définis par leur capacité à inhiber la formation des métastases sans affecter le développement de la tumeur primitive in vivo [5]. La fonction de GSM est validée par des expériences de surexpression de ces gènes dans des lignées tumorales hautement métastatiques, avec pour conséquence une réduction de leur potentiel métastatique testé dans des modèles expérimentaux, le plus souvent chez la souris. Des études cliniques corrélant la diminution d'expression des GSM dans les tumeurs humaines à certains indicateurs d'agressivité clinique confirment les données obtenues dans les modèles expérimentaux. II faut noter que les GSM ne sont pas toujours retrouvés dans les signatures métastatiques et qu'ils ne sont que rarement le siège de mutations ou de délétions. La diminution de leur expression est due le plus souvent à un mécanisme épigénétique, comme une méthylation du promoteur ou une modification post-transcriptionnelle et/ou traductionnelle [5]. Aujourd'hui, près de 12 GSM ont été décrits mais la liste n'est pas exhaustive.

\section{NM23}

Le produit du gène NM23 (NM23-HI chez l'homme ou NM23-MI chez la souris), découvert comme gène suppresseur de métastases dans une lignée de mélanome de souris, a été caractérisé peu après comme une nucléoside diphosphate kinase qui catalyse la synthèse des nucléosides triphosphates (NDPK, EC 2.7.4.6) [6]. Comme ce gène, maintenant appelé NME selon la nomenclature internationale, apparaît toujours dans la littérature sous le nom de NM23, nous garderons le nom d'origine dans cette revue. Depuis, d'autres gènes de cette famille ont été identifiés [7] dont les plus abondamment exprimés, NM23-H1 (NMEl) et NM23-H2 (NME2), codent des protéines identiques à $88 \%$. NME3 a été caractérisé par sa surexpression dans la phase aiguë des leucémies myéloïdes chroniques et NME4, identifié au laboratoire, code une isoforme spécifique des mitochondries. Les autres isoformes, plus éloignées phylogénétiquement, possèdent un ou plusieurs domaines NDPK en tandem, associés ou non à d'autres domaines comme la thiorédoxine. La plupart de ces isoformes sont associées aux structures axonémales et l'une d'entre elles est impliquée dans une pathologie ciliaire.

L'expression de NM23-HI est doublement associée à la cancérogenèse, et les observations suivantes ont été faites: (1) une surexpression précoce de NM23-H1 (et également de NM23-H2) dans les cellules tumorales de la plupart des tumeurs solides humaines par rapport au tissu sain, suggérant un rôle favorisant les étapes initiales de la progression tumorale, et (2), une perte de l'expression de NM23-Hl aux stades avancés de certains types de tumeurs comme les mélanomes, les tumeurs du sein, du côlon et des ovaires, et les carcinomes hépatocellulaires, inversement corrélée au potentiel métastatique [8]. Un rôle causal de NM23 dans la dissémination métastatique est démontré par: (1) une inhibition du potentiel métastatique de lignées tumorales agressives par l'expression forcée de NM23-H1 [2], et (2) une augmentation de l'incidence des métastases pulmonaires chez des souris doublement transgéniques, invalidées pour NM23-MI et développant des carcinomes hépatocellulaires résultant de l'expression hépato-spécifique de l'antigène T du virus SV40 [9]. En ce qui concerne le rôle de NM23H2/M2 dans le potentiel métastatique, les données de la littérature sont beaucoup moins abondantes et relativement discordantes.

Le (ou les) mécanisme(s) moléculaire(s) impliquant NM23 dans le contrôle du potentiel métastatique ne sont pas encore élucidés. Il était tentant de proposer que les NDPK, responsables de la synthèse du GTP, puissent jouer un rôle dans l'activation des protéines liant le GTP. Paradoxalement, il s'agit d'une inhibition, en accord avec le rôle de suppresseur de métastases. L'interaction de NM23-HI avec Tiaml, facteur d'échange nucléotidique de Racl, Rho-GTPase impliquée dans la motilité cellulaire, inhibe l'activation de Racl [10]. NM23-H1, en interagissant avec la protéine d'échaffaudage KSR, inhibe l'activation de la voie ERK/MAPK [11]. Une activité de type histidine kinase a été proposée pour NM23 [2]. Cependant, celle-ci n'a pas été démontrée pour la protéine recombinante purifiée et n'est pas compatible avec la structure tridimensionnelle de l'enzyme. Des travaux sur les mécanismes moléculaires de l'endocytose chez la drosophile et les cellules de mammifères suggèrent une coopération entre NM23 et la dynamine, GTPase indispensable à ce mécanisme, dans la modulation du signal induit par certains récepteurs, en particulier par le récepteur du FGF (fibroblast growth factor) [12]. Ainsi, l'absence de NM23 empêcherait le recyclage de ces récepteurs et prolongerait leur action. NM23 a également été impliquée dans la dynamique de l'attachement des cellules à la matrice extracellulaire via l'intégrine $\beta 1$ [13]. Une analyse transcriptomique comparative de lignées de carcinome surexprimant ou non NM23-HI et différant dans leur potentiel invasif, a permis d'identifier différents gènes dont l'expression est modulée par NM23-H1. II faut noter particulièrement une diminution induite par NM23-HI de l'expression de récepteurs membranaires, en particulier d'EDG2, récepteur du LPA (lipo- 


\begin{tabular}{|c|c|c|c|c|c|c|}
\hline Gène & $\begin{array}{c}\text { Nom } \\
\text { (Nom d'usage) }\end{array}$ & $\begin{array}{l}\text { Date de } \\
\text { décou- } \\
\text { verte }\end{array}$ & $\begin{array}{l}\text { Méthode } \\
\text { d'identi- } \\
\text { fication }\end{array}$ & $\begin{array}{l}\text { Localisation } \\
\text { chromoso- } \\
\text { mique }\end{array}$ & $\begin{array}{l}\text { Type de } \\
\text { tumeurs }\end{array}$ & Fonction \\
\hline $\begin{array}{l}\text { AKAP12 } \\
\text { (SSeCKS) }\end{array}$ & $\begin{array}{l}\text { A-kinase PRKA anchor } \\
\text { protein gravin } 12 \\
\text { (Src-suppressed C } \\
\text { kinase substrate) }\end{array}$ & 2001 & HS & $6 q 24-q 25$ & Prostate & $\begin{array}{l}\text { Inhibition de la formation des podosomes } \\
\text { Action anti-angiogénique }\end{array}$ \\
\hline BRMSl & $\begin{array}{l}\text { Breast cancer } \\
\text { metastasis } \\
\text { suppressor } 1\end{array}$ & 2000 & $\mathrm{TC}+\mathrm{DD}$ & $11 q 13-q 13.2$ & $\begin{array}{l}\text { Mélanome, } \\
\text { sein }\end{array}$ & $\begin{array}{l}\text { Communication cellule/cellule } \\
\text { (jonctions Gap) } \\
\text { Partenaire du complexe répresseur } \\
\text { transcriptionnel mSin3-HDAC }\end{array}$ \\
\hline $\begin{array}{l}\text { CD82 } \\
(\text { KAIl) }\end{array}$ & CD82 molecule & 1995 & HS & $11 p 11.2$ & $\begin{array}{l}\text { Prostate, } \\
\text { sein, } \\
\text { mélanome }\end{array}$ & $\begin{array}{l}\text { Atténuation de la voie de l'EGF-R } \\
\text { Inhibition de l'activation de c-Met et de Src } \\
\text { Interaction KAIl/DARC }\end{array}$ \\
\hline CRSP3 & $\begin{array}{l}\text { Cofactor required for } \\
\text { Spl transcriptional } \\
\text { activation subunit } 3\end{array}$ & 2003 & $\mathrm{TC}+\mathrm{MA}$ & $6 q 22.33-q 24.1$ & Mélanome & Régulateur de KiSSI \\
\hline $\begin{array}{l}\text { GDI2 } \\
\text { (RhoGDI2) }\end{array}$ & $\begin{array}{l}\text { GDP dissociation } \\
\text { inhibitor } 2\end{array}$ & 2002 & MA & $10 p 15$ & Vessie & Inhibition de l'activation de Rho-GTPases \\
\hline KISS1 & $\begin{array}{l}\text { KiSS-1 metastasis } \\
\text { suppressor }\end{array}$ & 1996 & $\mathrm{TC}+\mathrm{HS}$ & lq32 & $\begin{array}{l}\text { Mélanome, } \\
\text { sein }\end{array}$ & $\begin{array}{l}\text { KISSpeptines : Ligands de GPR54 } \\
\text { Répression transcriptionnelle de la MMP-9 }\end{array}$ \\
\hline $\begin{array}{l}\text { MAP2K4 } \\
(\text { MKK4) }\end{array}$ & $\begin{array}{l}\text { Mitogen-activated } \\
\text { protein kinase kinase } 4\end{array}$ & 1999 & $\mathrm{TC}+\mathrm{HS}$ & $17 p 11.2$ & $\begin{array}{l}\text { Prostate, } \\
\text { ovaire }\end{array}$ & Activation de p38 et de JNK \\
\hline $\begin{array}{l}\text { NDRGl } \\
(\operatorname{Drg}-1)\end{array}$ & $\begin{array}{l}N \text {-Myc downstream } \\
\text { regulated gene } 1 \\
\text { (Differentiation- } \\
\text { related gene } 1 \text { ) }\end{array}$ & 1997 & DD & $8 q 24$ & $\begin{array}{l}\text { Prostate, } \\
\text { côlon }\end{array}$ & Activation via MKK4 \\
\hline $\begin{array}{l}\text { NMEl } \\
(\text { NM23-Hl) }\end{array}$ & $\begin{array}{l}\text { Non-metastatic cells } 1 \\
\text { (Non-metastatic } \\
\text { clone } 23 \text { ) }\end{array}$ & 1988 & HS & $17 q 21.3$ & $\begin{array}{l}\text { Mélanome, } \\
\text { sein, } \\
\text { foie }\end{array}$ & $\begin{array}{l}\text { Activité nucléoside diphosphate kinase } \\
\text { Multifonctionnalité }\end{array}$ \\
\hline $\begin{array}{l}\text { PEBPl } \\
(\text { RKIP) }\end{array}$ & $\begin{array}{l}\text { Phosphatidylethanola- } \\
\text { mine binding protein } 1 \\
\text { (Raf kinase inhibitory } \\
\text { protein) }\end{array}$ & 2003 & MA & $12 q 24$ & $\begin{array}{l}\text { Prostate, } \\
\text { mélanome, } \\
\text { côlon, foie }\end{array}$ & $\begin{array}{l}\text { Inhibition de l'activation de ERK médiée } \\
\text { par Raf }\end{array}$ \\
\hline $\begin{array}{l}\text { TXNIP } \\
\text { (VDUPI) }\end{array}$ & $\begin{array}{l}\text { Thioredoxin } \\
\text { interacting protein } \\
\text { (Vitamin D } \\
\text { upregulated protein) }\end{array}$ & 2003 & $\mathrm{TC}+\mathrm{MA}$ & $\operatorname{lq} 21.1$ & Mélanome & $\begin{array}{l}\text { Inhibiteur de la thiorédoxine } \\
\text { Action anti-proliférative } \\
\text { et pro-apoptotique }\end{array}$ \\
\hline
\end{tabular}

Tableau I. Les différents gènes suppresseurs de métastases. HS : hybridation soustractive; DD : criblage différentiel; MA : microarray; TC : transfert de chromosome. Les noms d'usage sont utilisés dans cet article car ils sont le plus souvent cités dans la littérature.

phosphatidic acid), activateur des Rho-GTPases, et de MET, récepteur de I'HGF (hepatocyte growth factor) ou scatter factor, activateur de la transition épithéliale-mésenchymateuse [14]. Un mécanisme totalement différent pourrait impliquer la propriété de NM23-Hl et/ou H2 de lier l'ADN et leur activité désoxyribonucléase vis-à-vis de l'ADN simple brin [15]. Ainsi, NM23-Hl et -H2 régulent la transcription de différents gènes tels que $c$-myc, le gène de la MMP-2 et de la sous-unité A du PDGF (platelet derived growth factor). L'activité nucléase de NM23-Hl est impliquée dans l'apoptose induite par les lymphocytes T cytotoxiques. La granzyme A, importée dans la cellule 
cible, éventuellement une cellule tumorale, libère NM23-HI du complexe SET permettant son transfert dans le noyau où elle clive I'ADN et induit la mort cellulaire [16].

\section{Les autres gènes suppresseurs de métastases}

La plupart des GSM (Tableau I) agissent sur la signalisation intracellulaire via deux voies majeures, celle dépendante des protéines $G$ monomériques (NM23, RhoGDI2 et SSeCKS) et hétérotrimériques (KISSI et CRSP3), et celle dépendante des MAPK (NM23, MKK4, RKIP, Drg-1), elle-même contrôlée par des GTPases monomériques, ce qui pourrait impliquer des voies communes ou interactives encore mal connues (Figure 2).

Concernant la signalisation dépendante des protéines $G$, RhoGDI2 est un inhibiteur de la dissociation du GDP de Rho et Rac, Rho-GTPases ayant un rôle déterminant dans la migration cellulaire [17]. En maintenant ces protéines sous une forme inactive liée au GDP, RhoGDI2 contribuerait à inhiber la motilité cellulaire. De plus, ce GSM contrôle la prolifération des cellules endothéliales au cours de l'angiogenèse par l'axe endothéline/récepteur de l'endothéline. Le mécanisme par lequel SSeCKS inhibe la formation

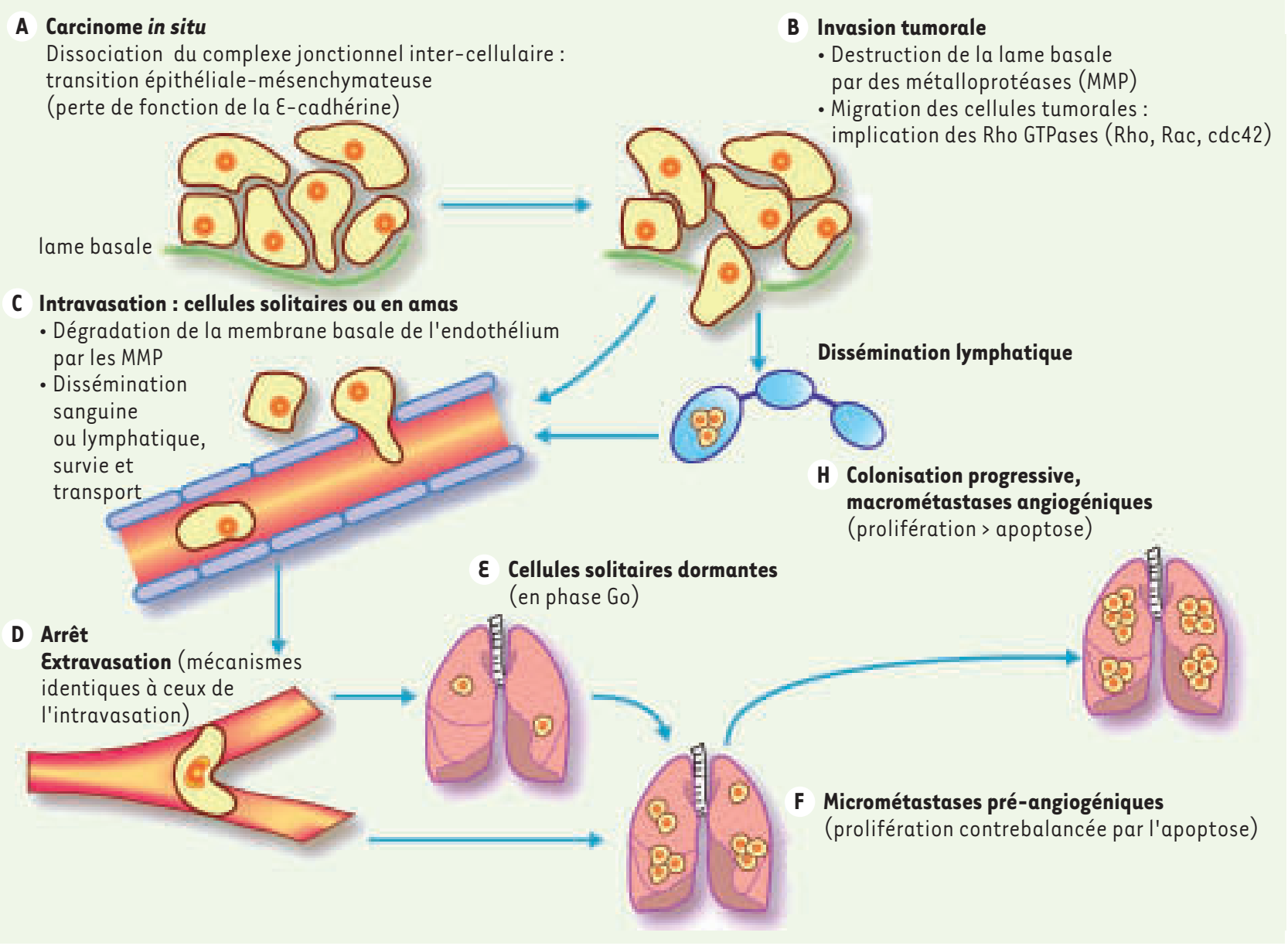

Figure 1. Les différentes étapes de la cascade métastatique (cas d'une tumeur épithéliale). Le détachement des cellules tumorales de la tumeur primitive épithéliale (carcinome) est en partie dû à l'altération de l'adhésion cellule/cellule, en particulier dépendante de la $\varepsilon$-cadhérine. Les deux fonctions majeures des cellules tumorales impliquées dans l'invasion tumorale sont la dégradation protéolytique de la lame basale assurée principalement par des métalloprotéases (MMP) et la migration cellulaire impliquant les Rho-GTPases. Après l'invasion du stroma péritumoral, les cellules tumorales traversent l'endothélium des vaisseaux sanguins ou lymphatiques (intravas ation) par dégradation de la membrane basale endothéliale grâce aux MMP. Elles sont ensuite transportées dans la circulation sanguine ou lymphatique à distance de la tumeur primitive jusqu'au niveau de l'endothélium de l'organe cible. La traversée de cet endothélium (extravasation) nécessite les mêmes propriétés des cellules tumorales que lors de la pénétration des structures vasculaires. Les cellules tumorales dans l'organe secondaire peuvent rester dormantes (en phase Go) pendant plusieurs mois ou années, ou proliférer d'emblée induisant la formation de micrométastases pré-angiogéniques (à l'index prolifératif et apoptotique élevés contrebalançant tout avantage prolifératif). La vascularisation des micrométastases pré-angiogéniques formant des macrométastases angiogéniques (ayant un avantage prolifératif) cliniquement détectables. 
des métastases est mal connu. II vient d'être rapporté que la ré-expression de SSeCKS dans des cellules NIH3T3 transformées par l'oncogène $S R C$ inhibait la formation des podosomes, structures riches en actine impliquées dans l'invasion tumorale et la migration, via les Rho-GTPases RhoA et cdc42, et restaurait la morphologie cellulaire [18]. L'équipe de Gelman propose aussi que SSeCKS puisse inhiber la formation des métastases en inhibant la production du VEGF, principal facteur pro-angiogénique, par les cellules tumorales et stromales, et en induisant la synthèse de facteurs solubles anti-angiogéniques [19]. Le gène KISSI code un précurseur, KiSS1, dont la protéolyse conduit à la formation des Kisspeptines. Les Kisspeptines sont les ligands du récepteur couplé aux protéines $G$ GPR54 et jouent un rôle majeur dans la régulation de l'axe gonadotrope en stimulant la sécrétion hypothalamique de GnRH. En ce qui concerne l'effet suppresseur de métastases de KISSI, les Kisspeptines sécrétées par les cellules tumorales pourraient agir sur les cellules tumorales par un mécanisme autocrine et/ou paracrine, et/ou sur les cellules stromales par un mécanisme paracrine via GPR54 ou d'autres récepteurs non définis à ce jour [20]. De plus, KISSl réprime la transcription du gène de la MMP-9, impliquée dans l'invasion tumorale, en inhibant la liaison de $\mathrm{NF}-\kappa \mathrm{B}$ sur le promoteur de ce gène [21]. Quant à CRSP3, sa surexpression dans des cellules humaines de mélanome entraîne une augmentation de l'expression de KISSI suggérant que KISS1 est régulé par CRSP3 [22]. VDUP1, un GSM en amont de KISS1, en interagissant avec la thiorédoxine (TXN), favoriserait l'activation de ASKl, une kinase proapoptotique [22]. L'équipe de D. R. Welch suggère une voie régulatrice des métastases $:$ CRSP3 $\rightarrow$ VDUPI $\rightarrow$ KISSI.

Concernant la voie des MAPK, les travaux d'Aguirre-Ghiso et al. montrent que l'équilibre entre l'activation de p38 et de $\varepsilon R K 1 / 2$ est déterminant dans l'état prolifératif ou dormant d'une tumeur ce qui pourrait influencer la colonisation métastatique au niveau du site secondaire [23]. L'activation de p38 induit une inhibition de la croissance tumorale alors qu'une activation de ERKl/2 facilite la dissémination tumorale. MKK4 active les kinases JNK et p38 alors que NM23 et RKIP inhibent la voie ERK1/2. L'équipe de Rinker-Schaeffer a récemment montré que selon le type de cellules tumorales, prostatique ou ovarien, l'effet suppresseur de métastases de MKK4 résultait de l'activation de JNK ou de p38, respectivement [24, 25]. Le mécanisme par lequel Drg-1 inhibe le potentiel métastatique n'est pas connu. Néanmoins, Drg-1 est activée via MKK4 suggérant qu'elle agirait en aval de MKK4 [26]. MKK4 pourrait être également activée par VDUPI via l'activation de la kinase ASKl déjà mentionnée. Enfin, RKIP, comme NM23, est un régulateur de la voie Ras/Raf/MEK/ERK dont la surexpression inhibe l'activation de $\varepsilon R K 1 / 2$ [27].
D'autres GSM modulent l'endocytose et les interactions cellule/cellule. Le produit du gène KAll est une glycoprotéine appartenant à la superfamille des tétraspanines, protéines de la membrane cellulaire à 4 domaines transmembranaires régulant la migration, l'invasion et la fusion cellulaire. La surexpression de KAll dans les cellules épithéliales mammaires HB2 induit une diminution de la migration cellulaire en réponse à l'EGF corrélée à une augmentation de l'endocytose du complexe EGF/EGF-R [28]. D'autres études rapportent que l'activité anti-métastatique de KAll pourrait être due à l'inhibition de l'activation de MET ou de la kinase Src [29]. Récemment, l'équipe de K. Watabe a montré que la fonction anti-métastatique de KAIl dépendait d'une interaction directe entre KAIl des cellules tumorales et le récepteur DARC présent à la surface des cellules endothéliales, induisant une inhibition de la prolifération et la sénescence des cellules tumorales, et ainsi la suppression des métastases [30]. En effet, l'injection intraveineuse de cellules de mélanome Bl6F10 surexprimant KAll à des souris invalidées pour DARC induit la formation de métastases pulmonaires alors que l'injection de ces mêmes cellules à des souris sauvages inhibe la dissémination métastatique, suggérant que la fonction anti-métastatique de KAll est dépendante de la présence de DARC. BRMS1, partenaire du complexe répresseur transcriptionnel mSin3-HDAC, pourrait inhiber la transcription de gènes pro-métastatiques. En particulier, BRMSl réprime la transcription de gènes cibles dont l'activation est NF- $\kappa B$ dépendante, notamment des gènes $U P A$ et $O P N$, impliqués dans l'invasion tumorale $[31,32]$. BRMS1 est aussi capable de maintenir les communications cellule/cellule au niveau des jonctions communicantes Gap [33]. Un autre gène, celui de la maspine, a une expression inhibée par la voie NF- $\kappa B$. II est connu comme suppresseur de métastases mais a également été rapporté comme suppresseur de tumeur [34].

Quelques nuances sont à apporter à l'ensemble de ces données. II semble que l'activité anti-métastatique de certains GSM dépende du contexte cellulaire et tissulaire. Dans certains cas, il semblerait, paradoxalement, que leur absence inhibe la dissémination métastatique ou que leur présence la favorise. Ainsi, NM23 est surexprimé dans les neuroblastomes les plus agressifs par rapport à ceux de stade précoce [35]. Une donnée récente rapporte que l'invalidation in vitro du gène MKK4 dans la lignée tumorale pancréatique PL5 inhibe la formation des métastases pulmonaires chez la souris nude [36]. Certains GSM seraient-ils aussi des gènes suppresseurs de tumeurs? En effet, $41 \%$ des souris invalidées pour le gène VDUPI développent des carcinomes hépatocellulaires par rapport aux souris sauvages qui n'en développent pas, suggérant que la perte de VDUPI faciliterait la formation de la tumeur primitive [37].

\section{Place des GSM dans la cascade métastatique}

D'après leur mécanisme d'action, il semble que chaque GSM puisse altérer une ou plusieurs étapes de la cascade métastatique. En effet, BRMSI agirait au niveau des étapes précoces lors du détachement de la tumeur primitive en maintenant les jonctions inter-cellulaires mais également, comme KISS1, sur l'invasion. NM23 agirait sur l'adhérence cellule/cellule et cellule/matrice extracellulaire et également, comme RhoGDI2 et SSeCKS, en inhibant la motilité des cellules tumorales via, entre autres, un 


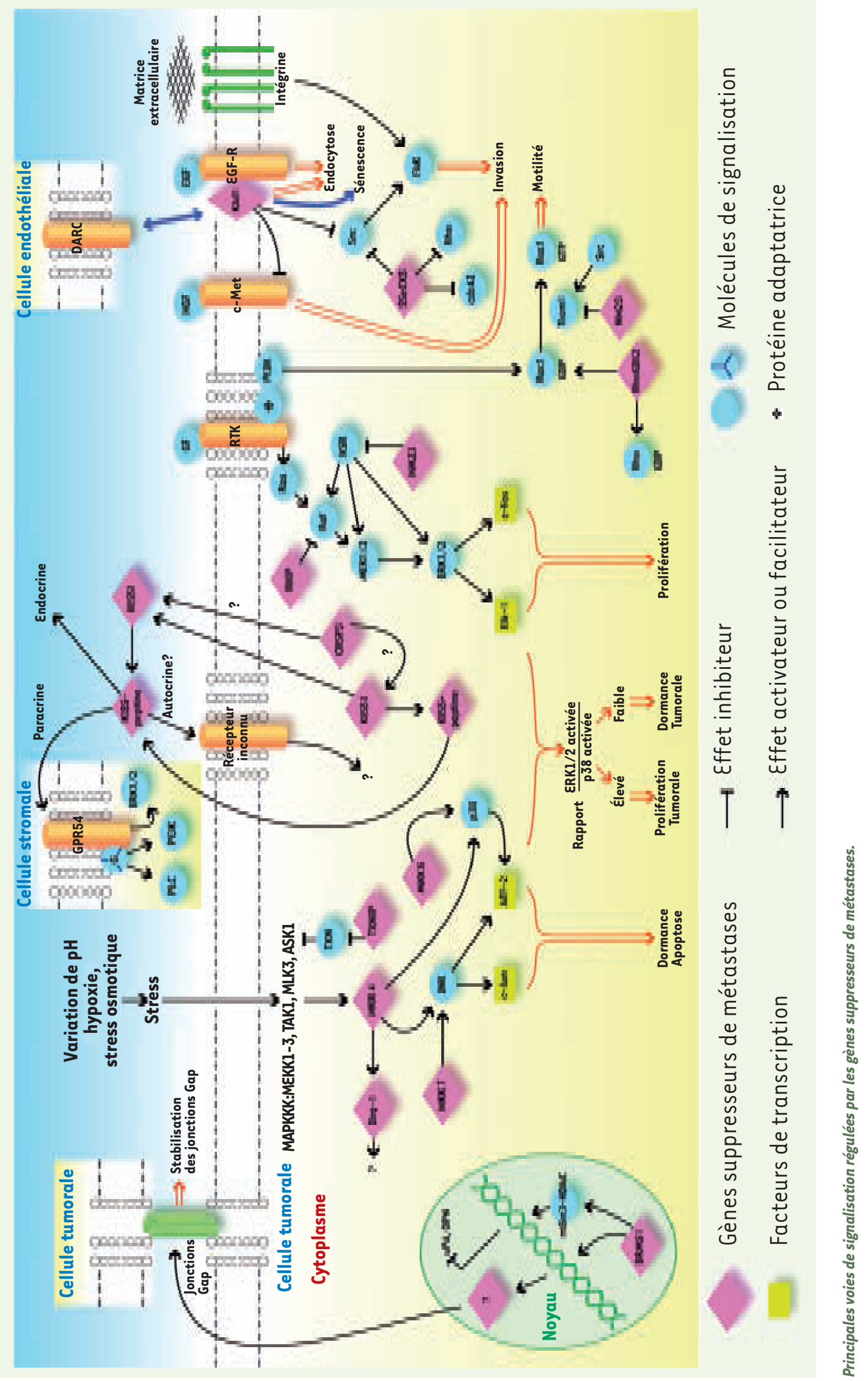


४ Figure 2. Principales voies de signalisation régulées par les gènes suppresseurs de métastases. Deux voies majeures sont régulées par les GSM, celle dépendante des MAPK et celle dépendante des protéines G. MKK4 active JNK et p38 alors que MKK6 et MKK7 activent p38 et JNK, respectivement. Drg-1 est activé via VDUP1. TXNIP active MKK4 via ASK1. NM23, en intéragissant avec KSR, et RKIP, par interaction avec Raf, inhibent l'activation de ERK1/2. Les protéines G monomériques et hétérotrimériques sont aussi la cible des GSM. RhoGD12 maintient les protéines Rho et Racl à l'état inactif liées au GDP. NM23 par interaction avec Tiaml inhibe spécifiquement l'activation de Racl. SSeCKS inhibe l'activation de RhoA et cdc42 et atténue le phénotype tumoral induit par l'oncogène Src. Les KISS-peptlines sécrétées par les cellules tumorales pourraient agir sur les cellules tumorales par un mécanisme autocrine et/ou paracrine et/ou sur les cellules stromales par un mécanisme paracrine via GPR54 ou d'autres récepteurs non définis à ce jour. CRSP3 régule l'expression de KISSI. KAIl et BRMSI modulent l'endocytose et les interactions cellule/cellule, respectivement. KAll facilite l'endocytose du récepteur de l'EGF et BRMSI renforce les jonctions intercellulaires. KAll pourrait également interagir avec la protéine DARC située à la surface des cellules endothéliales. GF : facteur de croissance; HGF : hepatocyte growth factor; EGF : epidermal growth factor; RTK : récepteur à activité tyrosine kinase ; MAPKKK : Map kinase kinase kinase ; uPA : urokinase-type plasminogen activator; OPN : ostéopontine [42].

mécanisme commun, l'inhibition de l'activation de RhoGTPases. KISS1 et MKK4 interviendraient lors de l'étape de colonisation en empêchant la croissance des cellules tumorales au niveau du site secondaire. En effet, l'équipe de D.R. Welch a montré que si le nombre de cellules de mélanome C8161.9 surexprimant ou non KISSI atteignant le poumon était comparable après injection intraveineuse, seules les cellules n'exprimant pas KISSI sont capables de former des métastases macroscopiques [20]. Cependant, cette étude ne peut exclure un rôle de KISSI au cours des étapes précoces de la cascade métastatique. CRSP3 et VDUPI, qui sont situés en amont de KISSI, devraient également contrôler l'étape de colonisation métastatique mais cela n'a pas été montré. L'activité anti-métastatique de MKK4 serait également due à une inhibition spécifique de la colonisation et non pas à une diminution du nombre de cellules tumorales arrivant au niveau du site secondaire. L'équipe de C. Rinker-Schaeffer a suggéré qu'un signal activant MKK4 serait spécifiquement présent au niveau de l'organe secondaire mais absent ou insuffisant au niveau de la tumeur primitive [24]. La fonction anti-métastatique de KAll, qui dépend d'une interaction entre KAIl des cellules tumorales et le récepteur DARC des cellules endothéliales, indique que KAII pourrait agir au niveau de la colonisation.

\section{Ré-exprimer les gènes suppresseurs de métastases : un défi thérapeutique}

Quelle est la valeur et l'intérêt des GSM en clinique? Une application potentielle serait leur utilisation comme marqueur du risque métastatique des tumeurs nouvellement diagnostiquées. II faut noter que la tumeur primitive est très hétérogène et il apparaît difficile de fonder une thérapie sur un prélèvement ne représentant que très partiellement l'intégralité de la tumeur. D'autre part, au moment du diagnostic de la tumeur primitive, la dissémination métastatique a déjà pu avoir lieu. Un des défis de la thérapie anti-métastatique serait d'augmenter l'expression des GSM dans les cellules déficientes ou de réactiver les voies dans lesquelles ils sont impliqués au niveau de la tumeur primitive ou du site secondaire. Deux approches sont envisageables: ré-exprimer le gène endogène à l'aide d'agents thérapeutiques ou apporter le gène déficient par thérapie génique. L'équipe de P. Steeg a mis en évidence dans le promoteur de NM23-HI une région potentiellement régulée par les stérö̈des et a observé que l'acétate de médroxyprogestérone (MPA), un agoniste des récepteurs des glucocorticoïdes, stimulait l'expression de NM23-HI et réduisait l'incidence, le nombre et la taille des métastases pulmonaires dans un modèle expérimental de métastases chez la souris nude [38]. De même, le traitement de souris avec un antagoniste de la voie endothéline impliquant RhoGDI2, l'atrasentan, réduit, de manière similaire à RhoGDI2, l'incidence des métastases pulmonaires dans un modèle murin [39]. L'équipe de D.R. Welch propose l'injection de Kisspeptines pour inhiber la colonisation métastatique [20]. II a été montré que l'injection intra-péritonéale d'un vecteur adénoviral exprimant NM23-HI réduit l'incidence des métastases hépatiques d'un modèle orthotopique de cancer de l'ovaire [40]. Très récemment, une équipe japonaise rapporte que l'administration intra-trachéale d'un vecteur adénoviral exprimant KAIl réduit de façon majeure la masse des métastases ganglionnaires d'un modèle orthotopique de cancer du poumon [41]. Ainsi, il paraît possible, tout du moins pour certains GSM, de restaurer leur fonction suppressive de métastases, offrant ainsi une réelle perspective thérapeutique anti-métastatique [43].

\section{Conclusions}

Le concept de « gène suppresseur de métastases » vient de l'identification de gènes capables de contrôler spécifiquement le potentiel métastatique des cellules tumorales sans affecter, le plus souvent, leur potentiel tumorigène. La métastatogenèse, bien que partageant certaines voies de la tumorigenèse, a un mécanisme propre comme le montre l'existence de gènes spécifiques favorisant ou inhibant la dissémination métastatique. Parmi ces gènes, les GSM agissent sur les propriétés intrinsèques des cellules tumorales et modulent, pour la plupart, des voies de signalisation contrôlées plus ou moins directement par des GTPases. L'analyse précise de ces voies devrait permettre l'identification de nouvelles cibles et de nouveaux agents thérapeutiques capables d'inhiber la formation des métastases. De même, une réactivation de leur expression endogène ou une stimulation des voies dans lesquelles ils sont impliqués pourrait constituer une thérapie anti-métastatique efficace apte à limiter, annihiler ou même prévenir la dissémination métastatique. $\diamond$ 


\section{SUMMARY}

NM23 and metastasis suppressor genes: update

Metastatic dissemination represents a leading cause of death in cancer patients. Elucidating the mechanisms of the metastatic process is therefore essential to control it. Since 1988, when the NME (NM23) gene was discovered, several genes specifically suppressing the metastatic potential of tumor cells, have been identified. These metastasis suppressor genes, which exhibit a reduced expression in metastatic tumor cells, are defined by their capacity to suppress metastatic dissemination in vivo without inhibiting primary tumor growth when transfected into metastatic cell lines and injected into experimental animals. Their decreased expression in a subset of human tumor cohorts is associated with a high metastatic potential, thus confirming the data obtained in experimental models. Most of these genes affect key signal transduction pathways, including mitogen-activated protein kinases, Rho-GTPases and G-protein-coupled receptors. These signaling categories control cell-cell and cell-matrix interactions, which are important in monitoring adhesion, invasion and migration properties of metastatic tumor cells. Reduced expression of metastasis suppressor genes is most often due to epigenetic mechanisms, suggesting that their re-expression could constitute a new anti-metastatic therapy. In this paper, we review the literature on metastasis suppressor genes, with a particular focus on NM23. $\diamond$

\section{GLOSSAIRE}

ASK1 : apoptosis signal-regulating kinase 1

DARC : Duffy antigen receptor for cytokines

EDG2 : endothelial differentiation, lysophosphatidic acid G-proteincoupled receptor 2

EGF : epidermal growth factor

FGF : fibroblast growth factor

GF : growth factor (facteur de croissance)

GnRH : gonadotropin-releasing hormone

HGF : hepatocyte growth factor

KSR : kinase suppressor of Ras

LPA : lysophosphatidic acid

MET : hepatocyte growth factor receptor

MAPKKK : MAP-kinase kinase kinase

MMP-2 : matrix metalloprotease-2

MMP-9 : matrix metalloprotease-9

mSin3-HDAC : mSin3-histone deacetylase

OPN : ostéopontine

PDGF : platelet-derived growth factor

RTK : récepteur à activité tyrosine kinase

UPA : urokinase-type plasminogen activator

VEGF : vascular endothelial growth factor

\section{RÉFÉRENCES}

1. Steeg PS, Bevilacqua G, Kopper L, et al. Evidence for a novel gene associated with low tumor metastatic potential. J Natl Cancer Inst 1988; $80: 200-4$.

2. Steeg PS. Metastasis suppressors alter the signal transduction of cancer cells. Nat Rev Cancer $2003 ; 3: 55-63$.

3. Christofori G. New signals from the invasive front. Nature $2006 ; 441: 444-50$.
4. Nguyen DX, Massague J. Genetic determinants of cancer metastasis. Nat Rev Genet $2007 ; 8: 341-52$.

5. Rinker-Schaeffer CW, O’Keefe JP, Welch DR, Theodorescu D. Metastasis suppressor proteins: discovery, molecular mechanisms, and clinical application. Clin Cancer Res 2006 ; 12 : 3882-9.

6. Wallet V, Mutzel R, Troll H, et al. Dictyostelium nucleoside diphosphate kinase highly homologous to $\mathrm{Nm} 23$ and Awd proteins involved in mammalian tumor metastasis and Drosophila development. J Natl Cancer Inst $1990 ; 82$ : 1199-202.

7. Lacombe ML, Milon L, Munier A, et al. The human Nm23/nucleoside diphosphate kinases. J Bioenerg Biomembr 2000 ; 32 : 247-58.

8. Boissan M, Lacombe ML. Nm23/NDP kinases in hepatocellular carcinoma. J Bioenerg Biomembr 2006 ; 38 : 169-75.

9. Boissan M, Wendum D, Arnaud-Dabernat $S$, et al. Increased lung metastasis in transgenic NM23-Null/SV40 mice with hepatocellular carcinoma. J Natl Cancer Inst $2005 ; 97: 836-45$.

10. Otsuki Y, Tanaka M, Yoshii S, et al. Tumor metastasis suppressor nm23Hl regulates Racl GTPase by interaction with Tiaml. Proc Natl Acad Sci USA $2001 ; 98: 4385-90$.

11. Salerno M, Palmieri D, Bouadis A, et al. Nm23-Hl metastasis suppressor expression level influences the binding properties, stability, and function of the kinase suppressor of Rasl (KSRl) Erk scaffold in breast carcinoma cells. Mol Cell Biol $2005 ; 25: 1379-88$.

12. Dammai V, Adryan B, Lavenburg KR, Hsu T. Drosophila awd, the homolog of human $\mathrm{nm} 23$, regulates FGF receptor levels and functions synergistically with shi/dynamin during tracheal development. Genes Dev $2003 ; 17: 2812-24$.

13. Fournier HN, Dupe-Manet $S$, Bouvard D, et al. Integrin cytoplasmic domain-associated protein lalpha (ICAP-lalpha) interacts directly with the metastasis suppressor $\mathrm{nm} 23-\mathrm{H} 2$, and both proteins are targeted to newly formed cell adhesion sites upon integrin engagement. J Biol Chem $2002 ; 277: 20895-902$.

14. Horak C $($, Lee JH, Elkahloun A, et al. NM23-H1 suppresses tumor cell motility by downregulating the LPA receptor. Cancer Res 2007 ; 67 : 7238-46.

15. Goswami S, Yoon J, Abramczyk B, et al. Molecular and functional interactions between Escherichia coli nucleoside-diphosphate kinase and the uracil-DNA glycosylase Ung. J Biol Chem 2006; 281 : 32131-9.

16. Fan Z, Beresford PJ, Oh DY, et al. Tumor suppressor NM23-Hl is a granzyme A-activated DNase during CTL-mediated apoptosis, and the nucleosome assembly protein SET is its inhibitor. Cell $2003 ; 112: 659-72$.

17. Gildea JJ, Seraj MJ, Oxford G, et al. RhoGDI2 is an invasion and metastasis suppressor gene in human cancer. Cancer Res 2002; 62: 6418-23.

18. Gelman IH, Gao L. SSeCKS/Gravin/AKAP12 metastasis suppressor inhibits podosome formation via RhoA- and Cdc42-dependent pathways. Mol Cancer Res $2006 ; 4: 151-8$.

19. Su B, Zheng $Q$, Vaughan MM, et al. SSeCKS metastasis-suppressing activity in MatLyLu prostate cancer cells correlates with vascular endothelial growth factor inhibition. Cancer Res 2006 ; 66 : 5599-607.

20. Nash KT, Phadke PA, Navenot JM, et al. Requirement of KISS I secretion for multiple organ metastasis suppression and maintenance of tumor dormancy. J Natl Cancer Inst 2007 ; 99 : 309-21.

21. Yan C, Wang H, Boyd DD. KiSS-1 represses $92-k$ Da type IV collagenase expression by down-regulating NF-kappa B binding to the promoter as a consequence of Ikappa Balpha -induced block of p65/p50 nuclear translocation. J Biol Chem $2001 ; 276: 1164-72$.

22. Goldberg SF, Miele ME, Hatta N, et al. Melanoma metastasis suppression by chromosome 6: evidence for a pathway regulated by CRSP3 and TXNIP. Cancer Res $2003 ; 63: 432-40$.

23. Aguirre-Ghiso JA, Estrada Y, Liu D, Ossowski L. ERK(MAPK) activity as a determinant of tumor growth and dormancy; regulation by p38(SAPK). Cancer Res 2003; 63 : 1684-95.

24. Vander Griend DJ, Kocherginsky M, Hickson JA, et al. Suppression of metastatic colonization by the context-dependent activation of the c-Jun NH2-terminal kinase kinases JNKK1/MKK4 and MKK7. Cancer Res $2005 ; 65: 10984-91$.

25. Hickson JA, Huo D, Vander Griend DJ, et al. The p38 kinases MKK4 and MKK6 suppress metastatic colonization in human ovarian carcinoma. Cancer Res $2006 ; 66: 2264-70$.

26. Motwani M, Sirotnak FM, She $Y$, et al. Drgl, a novel target for modulating sensitivity to CPT-11 in colon cancer cells. Cancer Res $2002 ; 62: 3950-5$.

27. Schuierer MM, Bataille F, Weiss TS, et al. Raf kinase inhibitor protein is downregulated in hepatocellular carcinoma. Oncol Rep 2006 ; 16: 451-6. 


\section{REMERCIEMENTS}

Nous remercions le Docteur Christèle Desbois-Mouthon et Adeline Wohlhuter pour leur lecture du manuscrit et $y_{v e s}$ Chrétien pour son travail infographique. Nous regrettons de n'avoir pu citer, faute de place, tous les auteurs ayant contribué à l'étude des GSM. Ce travail a été soutenu par l'Institut National de la Santé et de la Recherche Médicale (Inserm), la Fondation pour la Recherche Médicale (FRM), le Groupement des Entreprises Françaises dans la Lutte contre le Cancer (GEFLUC), l'Association pour la Recherche contre le Cancer $(A R C)$ et la Ligue Nationale contre le Cancer.

28. Odintsova $\varepsilon$, Sugiura T, Berditchevski F. Attenuation of $\varepsilon G F$ receptor signaling by a metastasis suppressor, the tetraspanin CD82/KAI-1. Curr Biol $2000 ; 10: 1009-12$.

29. Sridhar SC, Miranti CK. Tetraspanin KAII/CD82 suppresses invasion by inhibiting integrin-dependent crosstalk with c-Met receptor and Src kinases. Oncogene $2006 ; 25: 2367-78$.

30. Bandyopadhyay $S$, Zhan R, Chaudhuri A, et al. Interaction of KAll on tumor cells with DARC on vascular endothelium leads to metastasis suppression. Nat Med 2006; 12 : 933-8.

31. Cicek M, Fukuyama R, Welch DR, et al. Breast cancer metastasis suppressor 1 inhibits gene expression by targeting nuclear factor-kappaB activity. Cancer Res 2005 ; 65 : 3586-95.

32. Samant RS, Clark DW, Fillmore RA, et al. Breast cancer metastasis suppressor 1 (BRMS1) inhibits osteopontin transcription by abrogating NF-kappaB activation. Mol Cancer $2007 ; 6: 6$.

33. Saunders MM, Seraj MJ, Li Z, et al. Breast cancer metastatic potential correlates with a breakdown in homospecific and heterospecific gap junctional intercellular communication. Cancer Res 2001; 61 : 1765-7.
34. Luo JL, Tan W, Ricono JM, et al. Nuclear cytokine-activated IKKalpha controls prostate cancer metastasis by repressing Maspin. Nature 2007 ; $446: 690-4$.

35. Hailat N, Keim DR, Melhem RF, et al. High levels of $\mathrm{p} 19 / \mathrm{nm} 23$ protein in neuroblastoma are associated with advanced stage disease and with $\mathrm{N}$-myc gene amplification. J Clin Invest $1991 ; 88$ : 341-5.

36. Cunningham SC, Gallmeier $\varepsilon$, Hucl T, et al. Targeted deletion of MKK4 in cancer cells: a detrimental phenotype manifests as decreased experimental metastasis and suggests a counterweight to the evolution of tumor-suppressor loss. Cancer Res 2006; 66 : 5560-4.

37. Sheth SS, Bodnar JS, Ghazalpour A, et al. Hepatocellular carcinoma in Txnip-deficient mice. Oncogene $2006 ; 25: 3528-36$.

38. Palmieri D, Halverson D0, Ouatas T, et al. Medroxyprogesterone acetate elevation of $\mathrm{Nm} 23-\mathrm{HI}$ metastasis suppressor expression in hormone receptor-negative breast cancer. J Natl Cancer Inst $2005 ; 97: 632-42$.

39. Titus B, Frierson HF Jr, Conaway M, et al. Endothelin axis is a target of the lung metastasis suppressor gene RhoGDI2. Cancer Res $2005 ; 65$ : 7320-7.

40. Li J, Zhou J, Chen G, et al. Inhibition of ovarian cancer metastasis by adeno-associated virusmediated gene transfer of $\mathrm{nm} 23 \mathrm{Hl}$ in an orthotopic implantation model. Cancer Gene Ther $2006 ; 13: 266-72$.

41. Takeda T, Hattori N, Tokuhara T, et al. Adenoviral transduction of MRP-1/CD9 and KAII/CD82 inhibits lymph node metastasis in orthotopic lung cancer model. Cancer Res 2007 ; $67: 1744-9$.

42. Chabas D. L'ostéopontine, une molécule aux multiples facettes. Med Sci (Paris) 2005 ; $21: 832-8$.

43. Nasarre $P$, Constantin B, Drabkin HA, Roche J. Sémaphorines et cancer : état des lieux. Med Sci (Paris) $2005 ; 21: 641-7$

44. Desjardins SF, Berchiche YA, Haddad E, Heveker N. CXCR4, un récepteur de chimiokine aux multiples talents. Med Sci (Paris) 2007 ; $23: 980-4$.

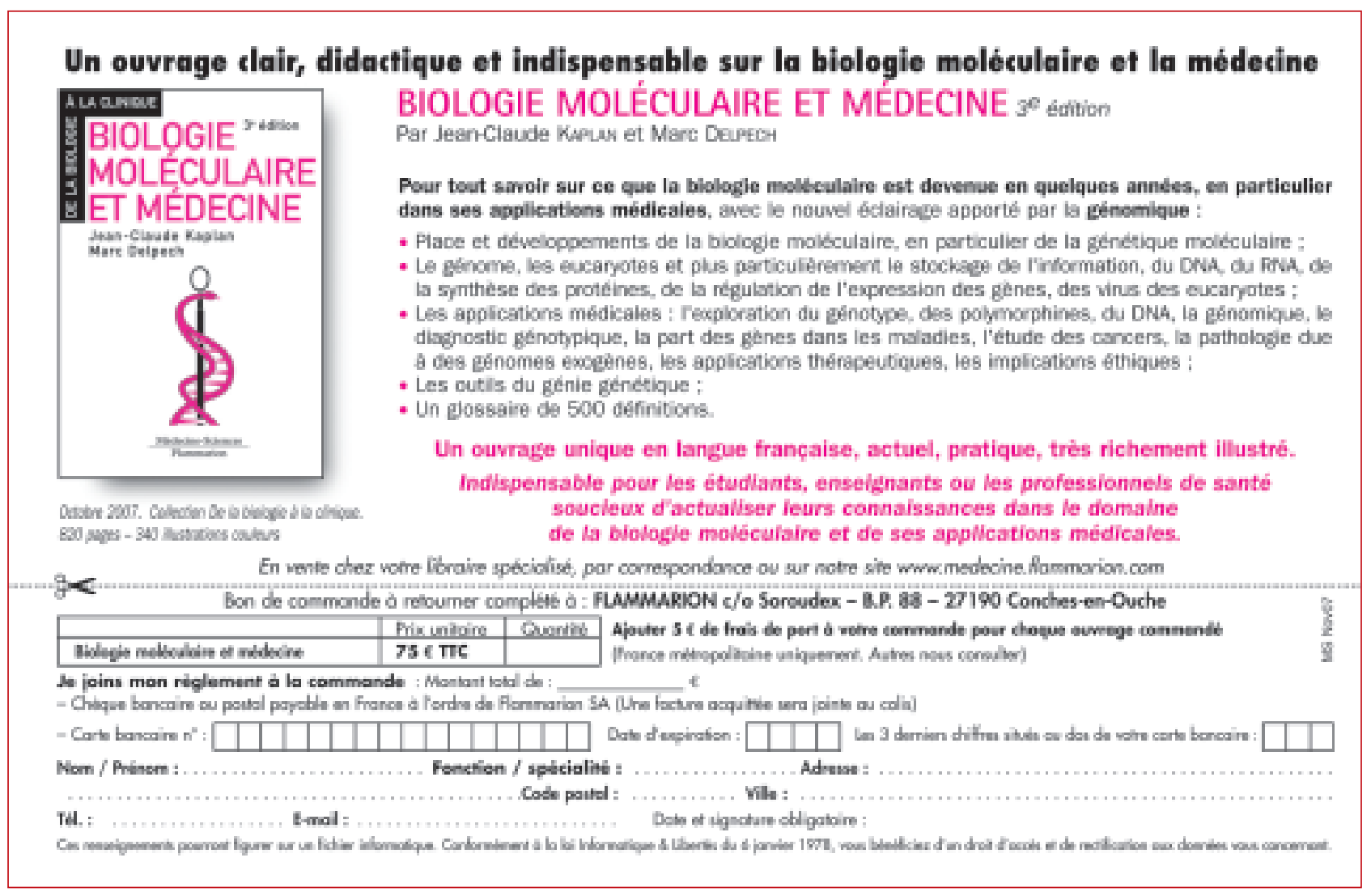

TIRÉS À PART

M.L. Lacombe

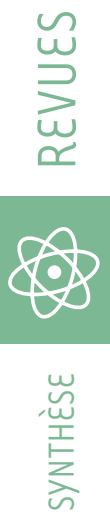




\section{Ateliers de formation 2008}

Renseignements et inscriptions

Ateliers de formation Inserm

101, rue de Tolbiac - 75654 Paris Cedex 13

Tél. : 33 (0)1 44236204 - Fax : 33 (0)1 44236293

ateliers@tolbiac.inserm.fr

\section{2}

\section{Modélisation physique} et analyse mathématique en biologie cellulaire

Organisateurs: Thierry Galli (Inserm/ Institut Jacques Monod, Paris), David Holcman (École Normale Supérieure, Paris)

Phase I - Le point sur...

13-14 mars 2008 - Saint-Raphaël

Nombre de participants : 80 maximum.

Conférenciers pressentis: Robert Austin (Princeton, USA), Daniel Choquet (France), Maité Coppey Moisan (France), Nicolas Destainville (France), Tuan Dinh, (Santa Barbara, USA), Michael Elbaum (Israel) Thierry Galli (France), Ari Helenius (Switzerland), David Holcman (France), Pierre-François Lenne (France), Karen Sachs (Stanford, USA), Stanislav Schvartsman (Princeton, USA).

Phase II • Maîtrise technique • Novembre 2008 • Paris/Toulouse Nombre de stagiaires : 12.

Date limite d'inscription : 25 janvier 2008

\section{3}

Théorie et outils biophysiques de mesure quantitative

Organisateurs: Danièle Altschuh (UMR CNRS/ULP 7175, Illkirch), Pierre Bongrand (UMR Inserm 600/CNRS 6212, Marseille)

Phase I - Le point sur...

10-11 avril 2008 - Saint-Raphaël

Nombre de participants : 80 maximum.

Conférenciers pressentis: Alain Brisson, (France), Gregory de Crescenzo (Canada) Hélène Feracci (France), Peter Hinterdorfer (Autriche), Steve Homans (UK), Deborah Leckband (Urbana, USA), Eric Perez (France) lacob Pielher (Germany), Frédéric Pincet (France), Pierre-Henri Puech (France), Pierre Schaaf (France), Gideon Schreiber (Israel), Olivier Thoumine (France), Cheng Zhu (Atlanta, USA)

Phase II • Maîtrise technique •

Mai-juin et sept.-oct. 2008 B Bordeaux/ Marseille/Paris/Strasbourg

Nombre de stagiaires : 2-6 par site.

Date limite d'inscription : 11 février 2008

\section{4}

\section{Modélisation}

et analyse statistique

des réseaux biologiques

Organisateurs: Emmanuel Barillot (Institut Curie, Paris), Stéphane Robin (AgroParisTech/INRA UMR0518, Paris), Jean-Philippe Vert (École des Mines, Paris)

Phase I - Le point sur...

15-16 mai 2008 • Saint-Raphaël

Nombre de participants : 80 maximum.
Conférenciers pressentis: Jacq Bernard, (France), Laurence Calzone (France), Florence d'Alché Buc (France), Hidde de Jong (France), Hervé Isambert (France), Alain Lilienbaum (France), Marcelline Kauffman (Belgique), Eugène Novikov (France), Ovidiu Radulescu (France), Korbinanian Strimmer (Allemagne), Denis Thieffry (France).

Phase II • Maîtrise technique •

Juin 2008 • Paris

Nombre de stagiaires: 20 .

Date limite d'inscription : 14 mars 2008

185

Protéines intrinsèquement désordonnées et pathologies associées : prédiction, caractérisation et fonction Organisateurs: Richard Haser (IBCP, Lyon), Sonia Longhi (AFMB, Marseille) Phase I - Le point sur...

19-20 mai 2008 - Saint-Raphaël

Nombre de participants : 80 maximum.

Conférenciers pressentis : Martin Blackledge (France), Keith Dunker (Indianapolis, USA) David Eliezer (New York, USA), Patrice Gouet (France), Sonia Longhi (France), Richard Kriwacki (Memphis, USA), Guy Lippens (France), Anne Poupon (France), Véronique Receveur-Bréchot (France), Peter Tompa (Hungary), Vladimir Uversky (Indianapolis, USA), Peter Wright (San Diego, USA).

Phase II - Maîtrise technique •

23-25 juin 2008 - Lyon

Nombre de stagiaires: 10.

Date limite d'inscription : 14 mars 2008

\section{6}

\section{Méthodes d'imagerie}

fonctionnelle in vivo:

de la molécule à la cellule

Organisateurs - Angela Giangrande, Michel Labouesse (IGBMC, Strasbourg)

Phase I - Le point sur...

5-6 juin 2008 •

Nombre de participants : 80 maximum.

Conférenciers pressentis: Jérôme Collignon

(France), Magdalena Goetz (Germany),

Stephen Hell (Germany), Thomas Lecuit

(France), Erez Raz (Germany), William

Schafer (UK), François Schweisguth (France),

Daniel St Johnston (UK), Antoine Triller

(France), Cornelius Weijer (UK), Joachim

Wittbrodt (Germany)

Phase II • Maîtrise technique •

Paris/Strasbourg •

15-19 septembre 2008 • Saint-Raphaël

Nombre de stagiaires : 3-4 par site.

Date limite d'inscription : 4 avril 2008

\section{7}

Analyse de la réplication ef de l'instabilité génétique par peignage moléculaire et autres approches "molécule unique "
Institut national

de la santé et de la recherche médicale
Organisateurs: Jean-Sébastien Hoffmann (IPBS, Toulouse), Philippe Pasero (IGH, Montpellier), Étienne Schwob (IGMM, Montpellier)

Phase I - Le point sur..

26-27 juin 2008 - Saint-Raphaël

Nombre de participants : 80 maximum.

Conférenciers pressentis: Aaron Bensimon (France), Michelle Debatisse (France), Olivier Hyrien (France), Dean Jackson (UK), Massimo Lopes (Switzerland), Raymond Monnat Jr (Seattle, USA), Paolo Norio (New York, USA), Marie-Jeanne Pillaire (France), Christophe Place (France), Yves Pommier (Bethesda, USA), Nick Rhind (Worcester, USA), JeanLouis Viovy (France).

Phase II - Maîtrise technique • Octobre 2008 Montpellier/Toulouse Nombre de stagiaires: 4 par site. Date limite d'inscription : 11 avril 2008

\section{8}

Défauts de transport

axonal et maladies

neurodégénératives :

stratégies pour comprendre

et traiter ces pathologies

Organisateurs : Thierry Galli (Inserm/ Institut Jacques Monod, Paris), Frédéric Saudou (Institut Curie, Orsay)

Phase I - Le point sur...

18-19 septembre 2008 • Saint-Raphaël Nombre de participants : 80 maximum.

Conférenciers pressentis: Patricia Bassereau (France), Fabrice Cordelières (France), Carlos Dotti (Belgique), Alexandra Dürr (France), Thierry Galli (France), Sandrine Humbert (France), Erika Holzbaur (Philadelphia, USA) John Kendrick-Jones (UK), Judith Melki (Israel), Chris Miller (UK), Frédéric Saudou (France), Giampietro Schiavo (UK), Tom Schwarz (Boston, USA), Michael Sendner (Germany), Kristen Verhey (Ann Arbor, USA).

Phase II • Maîtrise technique • Octobre/Novembre 2008 • Orsay/Paris Nombre de stagiaires : 6-8 par site.

Date limite d'inscription : 18 juillet 2008

\section{9}

Mutagénèse chimique in vivo et in vitro chez

la souris : récents progrès et applications pour la recherche de séries phénotypiques ou alléliques à haut débit Organisateurs: Philip Avner (Institut Pasteur, Paris), Véronique Blanquet (UGMA, Limoges), Yann Hérault (Institut de Transgénose, Orléans) Phase I - Le point sur...

2-3 octobre 2008 - Saint-Raphaël Nombre de participants : 80 maximum.

Conférenciers pressentis: Johan Auwerx, (France), Philip Avner (France), David Beier
(Boston, USA), Véronique Blanquet (France), Steve Brown (UK), Sophie Chantalat (France), Roger Cox (UK), Martin Hrabe de Angelis (Germany), lan Jackson (UK), Monica Justice (Houston, USA), George Kollias (Greece), John T. Kung (Taiwan), Juergen Laufs (Germany), Terry R. Magnuson (Chapel Hill, USA), John C. Schimenti (lthaca, USA), Karen L. Svenson (Bar Harbor, USA).

Phase II - Maîtrise technique • Novembre/Décembre 2008 Évryl Limoges/Orléans

Nombre de stagiaires : 4-6 par site. Date limite d'inscription : jer août 2008

\section{0}

Les récepteurs TLR : de la recherche à la médecine Organisateurs: Charles Hétru (IBMC, Strasbourg), Valérie Quesniaux (Institut de Transgénose, Orléans) Phase I - Le point sur...

16-17 octobre 2008 • Saint-Raphaël Nombre de participants : 80 maximum. Conférenciers pressentis: Julie Magarian Blander (New York, USA), Dominique Butoni-Gatel (France), Marco Colonna (St Louis, USA), Isabelle Couillin (France), Brian Foxwell (UK), Nick Gay (UK), François Huaux (Belgique), Jean-Luc Imler (France), Jean-Pau Mira (France), Jane A. Mitchell (UK), Muriel Moser (Belgique), Valérie Quesniaux (France), Jean-Claude Sirard (France), Mustapha SiTahar (France), Patrick Squiban (France), Philip Taylor (UK), François Trottein (France). Phase II - Maîtrise technique • 19-21 novembre 2008 - Orléans

Nombre de stagiaires : 8

Date limite d'inscription : 10 juillet 2008

\section{1}

Imagerie du petit animal : méthodes d'imagerie médicale pour l'exploration anatomique, fonctionnelle ef moléculaire in vivo

Organisateurs : Sylvie Chalon (Inserm U619, Tours), Marc Janier (Animage, Bron), Chantal Rémy (Grenoble Institut des Neurosciences, Grenoble)

Phase I - Le point sur...

20-21 octobre 2008 - Saint-Raphaël Nombre de participants : 80 maximum. Conférenciers pressentis: Claire Billotey (France), Irène Buvat (France), Jean-Luc Coll (France), Patrick Cozzone (France), Cyril Desvignes (France), Philippe Hantraye (France), Olga Millan (Spain), Laurent Monassier (France), François Moutou (France), Bertrand Tavitian (France), Annemie van der Linden (Belgique), Laurence Vico (France).

Phase II • Maîtrise technique • Octobre/Novembre 2008・Grenoblel Lyon/Marseille/Orsay/Tours

Nombre de stagiaires : 8-10 par site Date limite d'inscription : 8 août 2008 